\title{
From Infinitely Small to Infinitely Great
}

\author{
Aziz Ferradji \\ 37, Rue Larbi Ben M'hidi. Algiers. Algeria
}

\begin{abstract}
During his career in physics, Albert Einstein tried hard to elaborate a theory that allows to unify the quantum mechanics with the celestial mechanics, therefore he published the general relativity in order to establish a link between the special relativity and the Newton's gravitational law with the insight that gravity is not a force but rather a manifestation of curved space and time, which is produced by the mass-energy content of the space-time. But this theory has several flaws, especially when we try to explain the rotation of the moon around the earth, because we cannot represent a curved space created by the earth that allows the moon to gravitate, on the other hand Einstein did not accurately explain how the curved space is implicated in the mechanis $m$ of the earth's rotation around its axis that has the same direction as the earth's revolution around the sun, which makes it hard to explain the phenomenon of gravity with the general relativity, however it is obvious that gravity is the consequence of the creation of the universe, which means that the best way to define the origin of gravity, is the study of the transition from infin itely small to infin itely great.
\end{abstract}

Keywords Photons, Waves, Energy, Mass, Gravity, Atoms, Globes, Speed of light

\section{Introduction}

During his career in physics, Albert Einstein tried hard to elaborate a theory that allows to unify the quantum mechanics with the celestial mechanics, therefore he published the general relativity in order to establish a link between the special relativity and the Newton's gravitational law with the insight that gravity is not a force but rather a manifestation of curved space and time, which is produced by the mass-energy content of the space-time.

But this theory has several flaws, especially when we try to exp lain the rotation of the moon around the earth, because we cannot represent a curved space created by the earth that allows the moon to gravitate, on the other hand Einstein did not accurately explain how the curved space is implicated in the mechanis $m$ of the earth's rotation around its axis that has the same direction as the earth's revolution around the sun, which makes it hard to explain the phenomenon of gravity with the general relativity, however it is obvious that gravity is the consequence of the creation of the universe, which means that the best way to define the origin of gravity, is the study of the transition from infinitely small to infinitely great.

\section{The Creation of the Universe by the Light'S Energy}

* Corresponding author:

ferrazy179@yahoo.fr (Azi z Ferradji)

Published online at http://journal.sapub.org/ijtmp

Copyright (C) 2012 Scientific \& Academic Publishing. All Rights Reserved
According to the big bang theory, all materials existed as light's energy before the creation of the universe, on the other hand Thomas young stated that the light exists as waves that travel at a high speed in space, a century later, Albert Einstein postulated that the light is composed of particle-like small packets of energy called commonly photons, however if we contemplate the aspect of planets (infinitely great) as well as atoms and electrons (infinitely small), we would notice two major characteristics:

1. The spherical form.

2. The rotational movement.

Thus, if we consider that photons were implicated in the synthes is of atoms, electrons, and planets, then we would be forced to add the second characteris tic into the description of a beam of light, from which we hypothesize that photons move in a helical form which gives the beam of light a spiral aspect, however if we project this form on a plan we would get the same figure of the beam of light that was described by Thomas young, in other words, photons move as waves if the light is studied on a plan, but from a three-dimensional figure, photons move as a helix, To this end, the theory of the creation of the universe must be based on the fact that materials were created by photons that have the following characteristics :

-The high speed of the helical movement: $\mathrm{C}$

-Wavelength: $\lambda$

-Diameter of the helical movement: D

Also, if we study the geometrical form of a beam of light on a plan, we would notice that the linear speed of light in space varies according to an angle $(\sigma)$ that reflects the inclination of the wave, which means that the linear speed SL is determined by the projection of the helical speed's vector 
$\mathrm{C}$ on the axis of the light's propagation, according to the following figure:

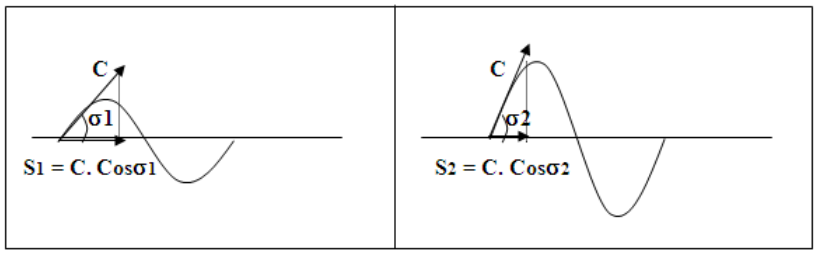

Figure 1. The impact of the variation of the angle $\sigma$ on the linear speed SL

From this figure we deduce that SL equals C. $\operatorname{Cos} \sigma$, that is to say when $\sigma 1$ is inferior to $\sigma 2$, then SL1 is higher than SL2.

The variation of the linear speed of the beam of light will have an impact on the characteristics of planets and atoms which will be explained in the next paragraphs.

\section{The Evaluation of the Photon'S Energy}

In 1900, Max Planck had published the formula $E=h v$ which indicates that the energy of light is inversely proportional to its wavelength, but if we introduce the notion of the angle $\sigma$, we would conclude that its variation has an effect on the amount of the light's energy as well.

When $\sigma$ is constant and $\lambda$ varies:

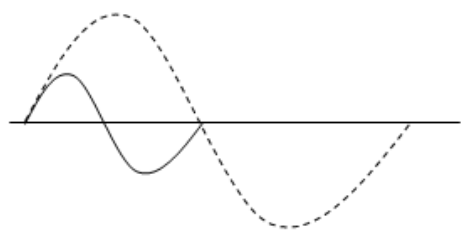

Figure 2. When $\sigma$ is const ant and $\lambda v$ aries

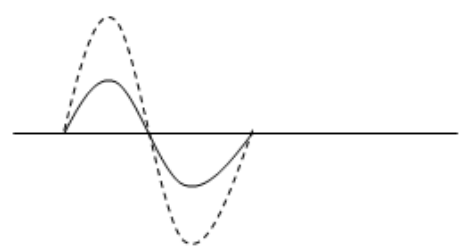

Figure 3. When $\lambda$ is constant and $\sigma$ varies

We can assimilate the elevation of $\lambda$ and $\sigma$ to the stretching of a rope that induces the decrease of the matter's amount per a length's unit, this indicates that the increase of the wavelength and the angle is the origin of the fragmentation of the beam of light which concords with the concept of Albert Einstein that the light is composed of infinitely s mall fragments.

From this, we deduce that the amount of those fragments reflects the energy of the light, which is proportional to the linear speed of light (according to $\operatorname{Cosines} \sigma$ ) and inversely proportional to the wavelength $\lambda$.

$$
\mathrm{E}=\frac{\mathrm{C} \cdot \operatorname{Cos} \sigma \cdots 1}{\lambda} \text { (the unit of } \mathrm{E} \text { is joule if it is multiplied }
$$
by Planck constant h)

\section{The Synthesis of Materials from the Light'S Energy}

The typical process that clarifies the production of materials by the light's energy is the photosynthesis that is effected by plants, and based on the absorption of the light by electrons, which leads systematically to the slowdown of the speed of light and to the concentration of photons according to the following equivalence:

\section{One photon $\rightarrow$ C. Cos $\sigma$}

Concentrated photon $\rightarrow$ Speed SL inferior than

\section{C.Cos $\sigma$}

On the other hand, we can hypothesise that the condensation of photons in space is based on their convergence into one single point that can be assimilated to a swirlof photons, wh ich leads to the appearance of a spherical material that rotates around its axis due to the helical characteristic of the beam of light that participates in the process of condensation.

Hence, the appearance of the concentrated photons is expressed by the variation of the following photon's parameters:

$\rightarrow$ The decrease of the linear speed.

$\rightarrow$ The decrease of the wavelength by the compaction of the ray.

$\rightarrow$ The elevation of the amount of the energy.

$\rightarrow$ The conversion of the energy into the mass according to Einstein's formula $E=m \cdot c^{2}$.

From a geometrical point of view, the concentration of photons can be represented by the following figure (the sphere is represented as a circle if it is projected on a plan) :

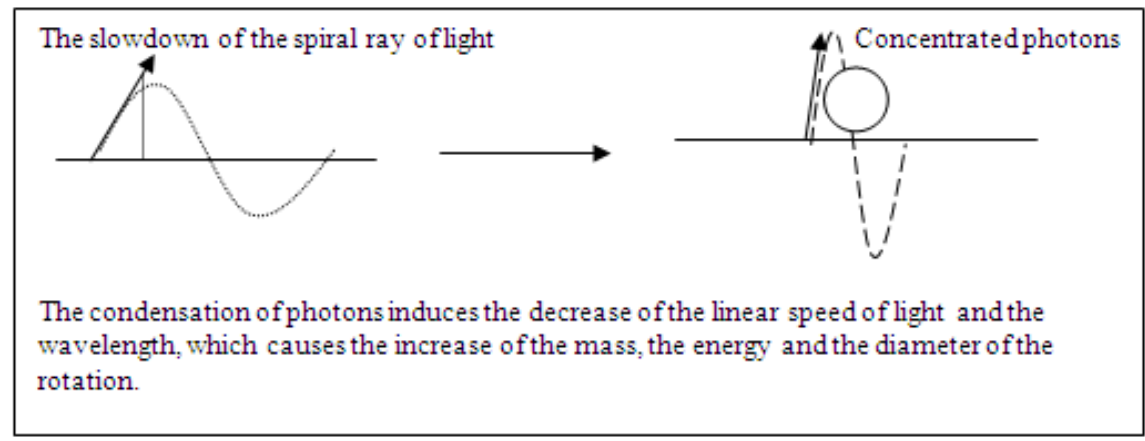

Figure 4. The condensation of photons induces the decrease of the linear speed of light and the wavelength, which causes the increase of the mass, the energy and the diameter of the rotation 
According to this figure, we deduce that the decrease of the speed is due to the enhancement of the angle $\sigma$ leading to the increase of its tangent, which means that the diameter of the helical movement is enhanced.

If we place a virtual observer in front the beam of light, we can mathematically explain the increase of the diameter by the elevation of the tangent $\sigma$

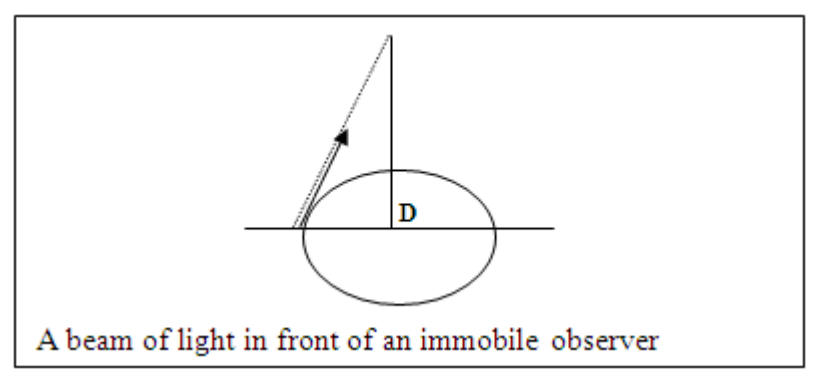

Figure 5. A beam of light in front of an immobile observer

According to the data presented above, we come to the conclusion that the mass and the energy are inversely proportional to the linear speed.

\section{Calculation of the Mass of the Concentrated Photons According to the Speed}

When the linear speed decreases from C.Cos $\sigma$ to SL the mass increases according to the following formula:
$\mathbf{M}=\frac{\mathbf{C} \cdot \operatorname{Cos} \sigma \cdots \cdots \cdot \mathbf{2}^{2}-}{\mathrm{SL}}$ (the unit of $\mathrm{M}$ is the number of photons)

When: $\mathrm{SL}=\mathrm{C} \cdot \operatorname{Cos} \sigma \rightarrow \mathrm{M}=$ one photon

$\mathrm{SL}=0 \rightarrow \mathrm{M}=$ infinite of concentrated photons

The formula 2 reflects a mathematical function that can be extrapolated as follows:

We consider: $\mathrm{SL}=\mathrm{C} \cdot \operatorname{Cos} \sigma-\alpha \mathrm{C} \cdot \operatorname{Cos} \sigma \quad(\alpha$ varies from 0 to 1 )

We have :

$$
\mathrm{M}=\frac{\mathrm{C} \cdot \operatorname{Cos} \sigma}{\mathrm{C} \cdot \operatorname{Cos} \sigma-\alpha \mathrm{C} \cdot \operatorname{Cos} \sigma}=\frac{1}{1-\alpha}
$$

$\mathrm{M}=1 / 1-\alpha \ldots . .3$

The formula 3 is a function which curve is represented in the following figure :

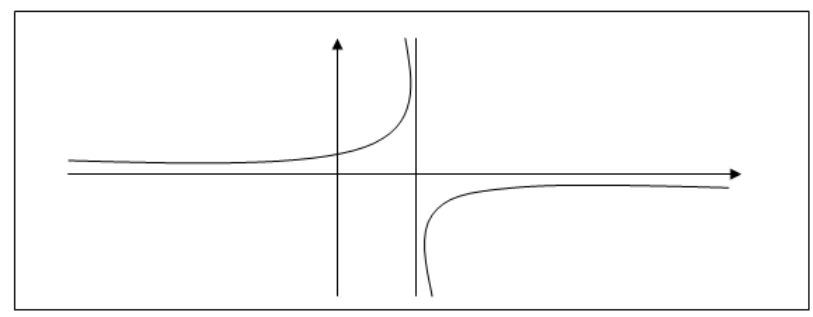

Figure 6. The curve of the formula: $M=1 / 1-\alpha$

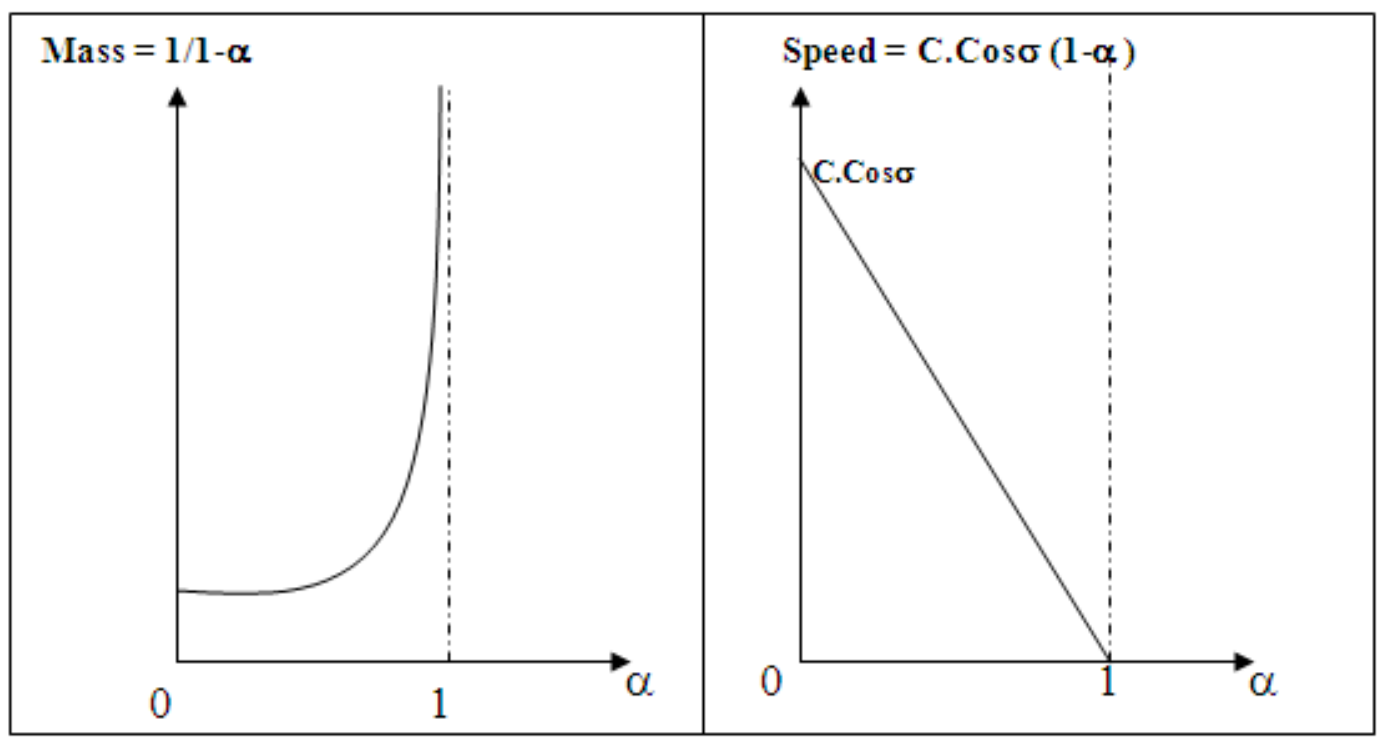

Figure 7. In this function $\alpha$ is defined between 0 and 1, the increase of the matter reflects the decrease of the Speed 


\section{The Characteristics of the Concentrated Photons}

The consequence of the convergence of photons that have a helical movement is the appearance of materials with a spherical form, which rotate around their axis and also acquire a speed that is lower than the photons' linear speed that participated in the formation of the matter, also, the helical speed of light $(\mathrm{SH})$ is reduced by the projection of its vector caused by the elevation of $\sigma$ (figure 6 )

Those materials are also likely to converge with each other, if they have the same diameter of the helical movement, and this will engender the continuing decrease of $\lambda, \mathrm{SL}, \mathrm{SH}$ and the increase of the mass M, the energy E and the diameter D.

The evolution of the vector's value reflects the transition from infinitely s mall to infin itely great, because the collision of different spherical materials will engender the decrease of the linear speed, until its annulment.
When $\mathrm{SL}=0$, the spherical material is defined by the following data:

$\rightarrow$ The mass tends to infin ite according to formula 3 .

$\rightarrow$ The spherical mass rotating around its axis.

$\rightarrow$ The angle $\sigma$ tends to $90^{\circ}$ which is responsible of the annulment of SL.

$\rightarrow$ Tangent of the angle $\sigma$ tends to infinite, which means that the diameter is great

$\rightarrow \lambda$ tends to zero which indicates that the helical movement becomes circular.

Those particularities concord perfectly with the characteristics of planets, from which we deduce that those planets where formed by the convergence of small spherical materials that have helical movements corresponding to gravity, in other words gravity is the consequence of the condensation of materials according to the formula 3 , which means that gravity is a speed with a curved tract, and this can be demonstrated if we place an immobilized observer in space:

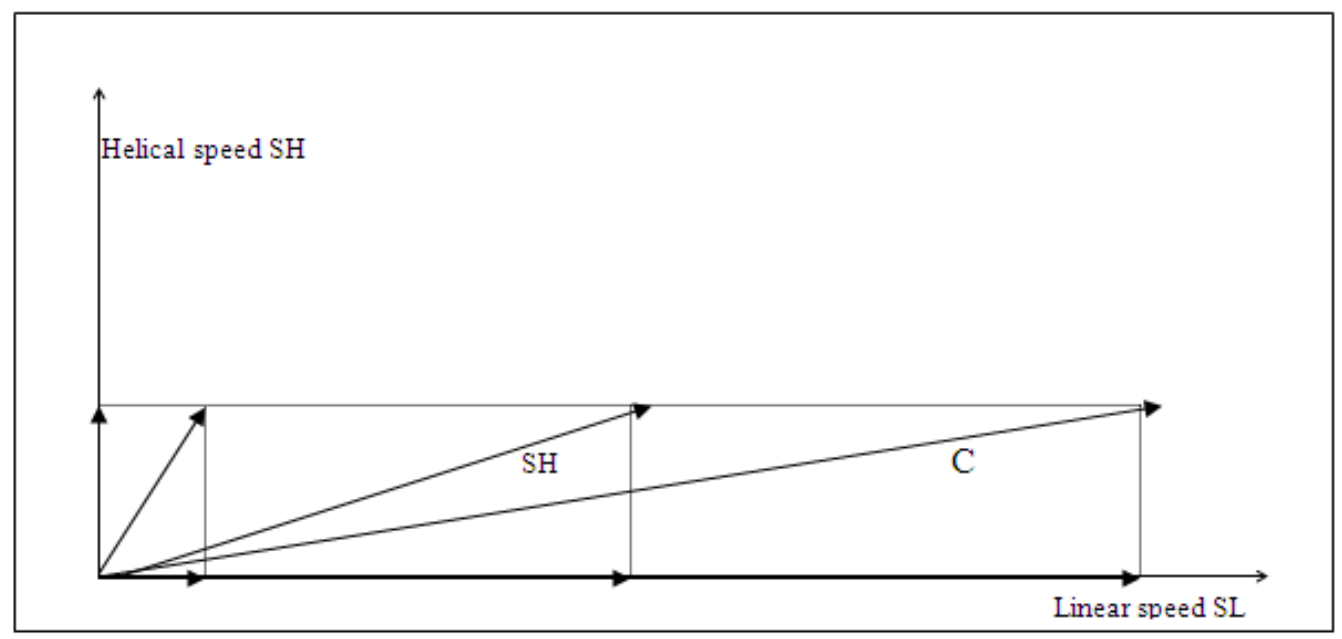

Figure 8. The evolution of the helical speed of light $\mathrm{C}$ to the circular speed of the planets

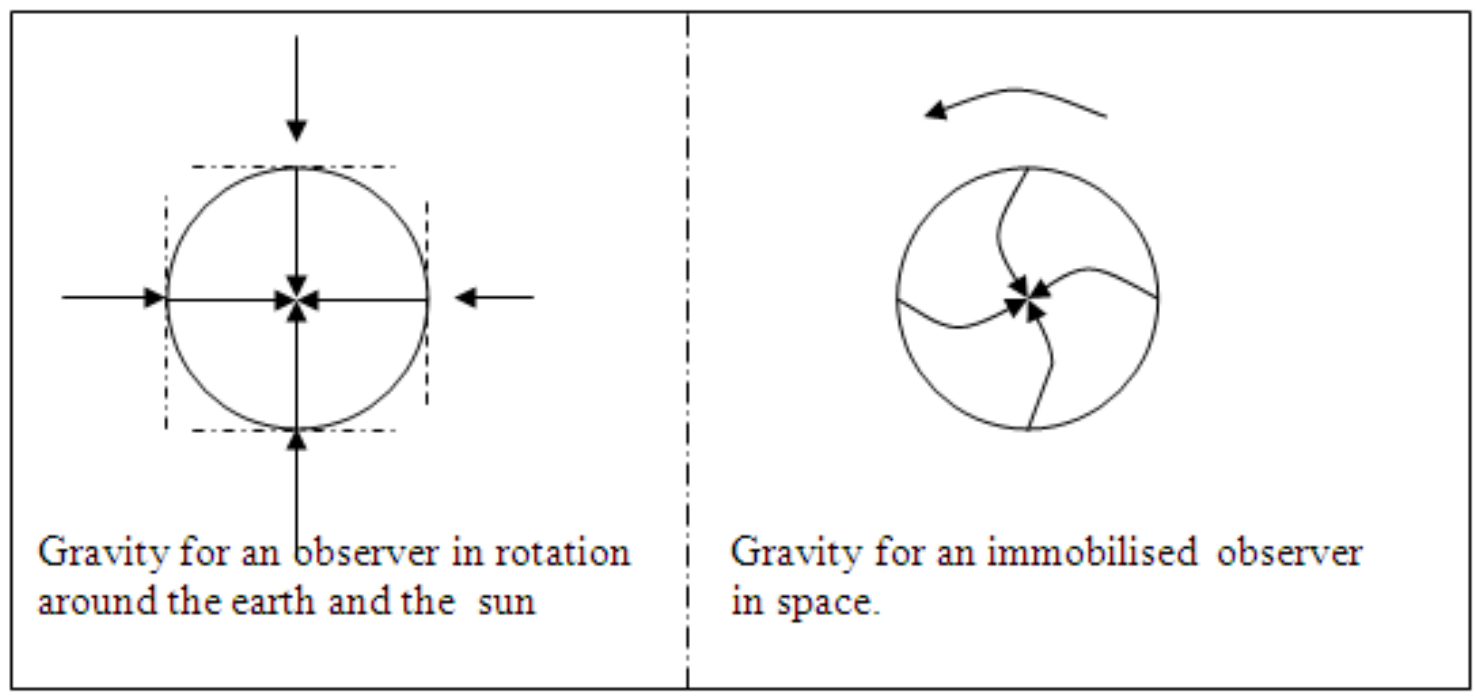

Figure 9. Gravity for an observer in rotation around the earth and the sun. Gravity for an immobilised observer in space 
This figure explains that if we place an observer on the earth that rotates around its axis and the sun, he would notice that the free-fall of objects is perpendicular to the tangential line of the earth's curvature, and also he would realize that the earth is immobile because the observer rotates around the earth and the sun as well, however, if the observer is immobile in space he would notice that the free-fall of objects is curved due to the rotational movement of the earth.

The second observation gives us the irrefutable evidence that gravity is the consequence of a swirl of the earth's particles due to the convergence of their helical movements that led to the appearance of a rotating spherical material, in other words, gravity is the driving force of the earth's rotation.

From this it might be deduced that the formation of the planets was the consequence of a series of concentrations that induced the conversion of the light's energy into the mass.

\section{The Calculation of the Concentrated Energy}

We have $\mathrm{M} 1=\frac{\mathrm{C} \cdot \operatorname{Cos} \sigma}{\mathrm{SL} 1}=\frac{1}{1-\alpha .1} \cdots$ That will be multip lied by formula 1

$$
\mathrm{E} 1=\mathrm{M} 1 \cdot \frac{\mathrm{C} \cdot \operatorname{Cos} \sigma}{\lambda} \quad \mathrm{E} 1=\frac{(\mathrm{C} \cdot \operatorname{Cos} \sigma)^{2}}{\mathrm{SL} 1 \cdot \lambda} \cdots \cdots \cdot 4
$$

This concentrated particle M1 is likely to converge with other particles that rotate at a same diameter in order to form another spherical material which mass M2 is expressed according to its decreased linear speed SL2:

$$
\mathrm{M} 2=\frac{\mathrm{C} \cdot \operatorname{Cos} \sigma}{\mathrm{SL} 2}=\frac{\mathrm{C} \cdot \operatorname{Cos} \sigma \cdot \mathrm{SL} 1}{\mathrm{SL} 1 \cdot \mathrm{SL} 2}
$$

$\rightarrow$ SL1/SL2 $=(1 / 1-\alpha 2)=$ Mo (the number of particles with mass M1 that formed M2)

$\rightarrow \mathbf{M} 2=\mathrm{Mt} \quad($ total mass of photons $)$

$$
\mathrm{M} 2=\frac{\mathrm{C} \cdot \operatorname{Cos} \sigma}{\mathrm{SL} 1} \cdot \frac{1}{1-\alpha .2}
$$

In this formula SL1 represents the gravitational speed ( linear) GL at which concentrated particles M 1 formed the spherical material M2 :

$$
\mathrm{M} 2=\frac{\mathrm{C} \cdot \operatorname{Cos} \sigma}{\mathrm{GL}} \cdot \frac{1}{1-\alpha .2}
$$

Fro $m$ this we deduce the value of E2 :

$$
\mathrm{E}_{2}=\frac{(\mathrm{C} \cdot \operatorname{Cos} \sigma)^{2}}{\mathrm{GL} \cdot \lambda} \cdot \frac{1}{1-\alpha 2}
$$

The total energy of materials formed by the concentrations of photons is :

$$
E=\frac{M_{0}(C \cdot \operatorname{Cos} \sigma)^{2}}{G L \cdot \lambda} \cdots \cdot 5 \text { (the unit is joule if } E \text { is }
$$

\section{multiplied by $h$ )}

This formula indicates that the energy of spherical materials ( planet, atom ...) is proportional to the amount of particles Mo that participate in the formation of the sphere, and inversely proportional to the linear gravity GL, the wavelength $\lambda$ and $\sigma$.

The rotational characteristic of planets is due to the gradual conversion of the helical speed of light (C)to the helical speed of gravity GH that turns to the revolution's speed of planets RS :

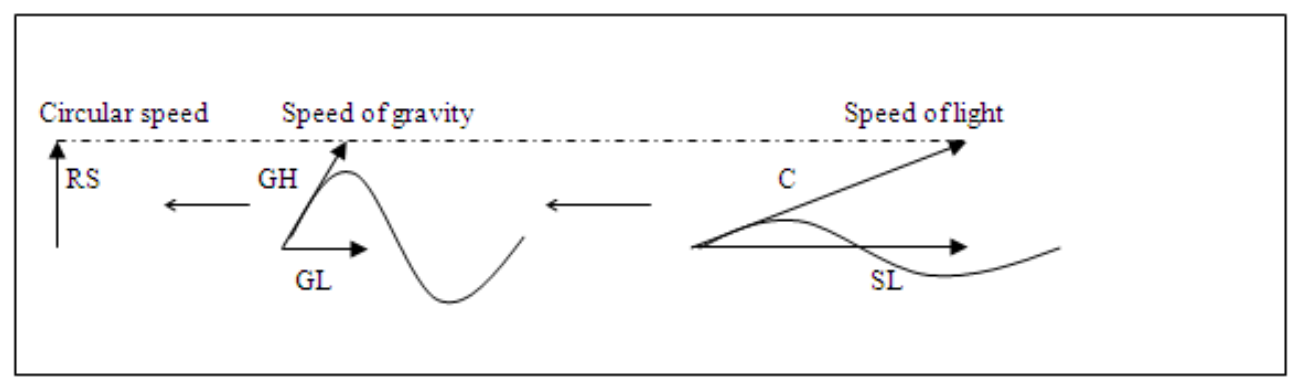

Figure 10. The conversion of the speed of light into the speed of gravity into the circular speed

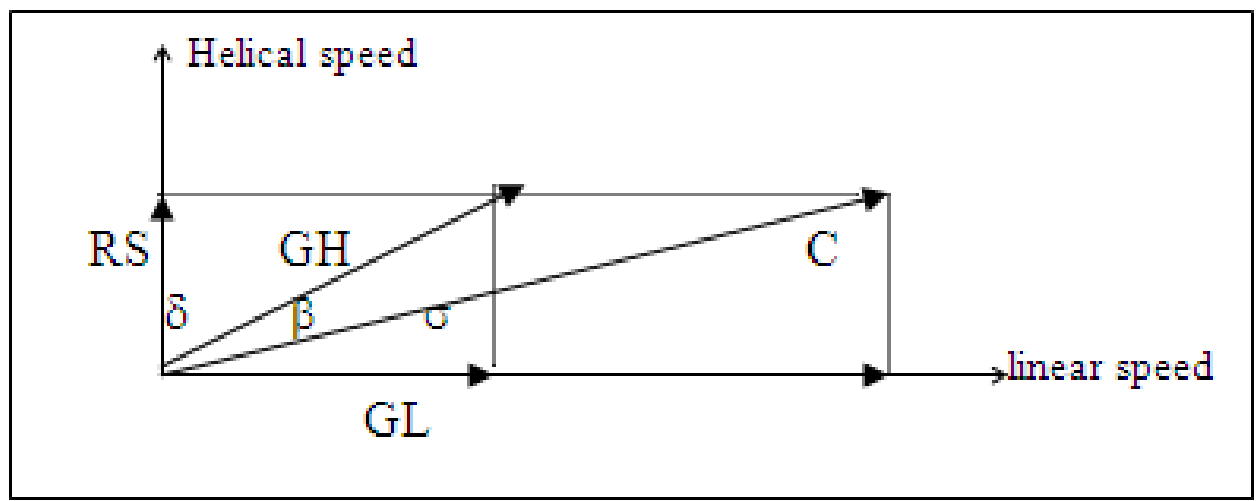

Figure 11. $\mathrm{GL}=\mathrm{GH} \cdot \operatorname{Cos}(\sigma+\beta)$ and $\mathrm{RS}=\mathrm{GH} \cdot \operatorname{Cos} \delta$ 
This figure indicates that the revolution's speed of planets $\mathrm{RS}$ is determined by the projection of GH's vector on the axis of the helical speed $\mathrm{SH}$, knowing that $\mathrm{GH}$ is the helical gravity that expresses the swirl of particles in the spherical materials ( planets, atoms..).

Thus, according to the figure 9 we have:

$$
\begin{aligned}
& \mathbf{G L}=\mathbf{G H} \cdot \operatorname{Cos}(\sigma+\beta) \\
& \mathbf{R S}=\mathbf{G H} \cdot \operatorname{Cos} \delta
\end{aligned}
$$

We realize that the concentration of photons which $\sigma$ tends to zero, leads to the annulment both linear and rotational speed RS which reflects an immobilized planet, This corresponds to the characteristics of the sun, and according to the formula 5 we deduce that gravity is too low on the surface of the sun.

\section{The Explanation of the Planet'S Ellipsoid Rotation}

If we place an observer in front of the direction of the beam of light that is submitted to condensation of photons (transition from infinitely small to infinitely great) we would get the following representation (figure 10):

From this figure we understand that the ellipsoid movement of planets is due to the ellipsoid helical movements of photons that were implicated in the synthes is of the matter.

\begin{tabular}{|l|l|l|}
\hline Photons & Concentrated photons \\
$\begin{array}{l}\text { - High linear Speed of light } \\
\text { - Wavelength } \lambda\end{array}$ & $\begin{array}{l}\text { - Reduced linear speed of light } \\
\text { - Reduced } \lambda\end{array}$ \\
- helix with a small $\sigma$
\end{tabular}

Figure 12. The transition from photons to atoms to planets according to their speed

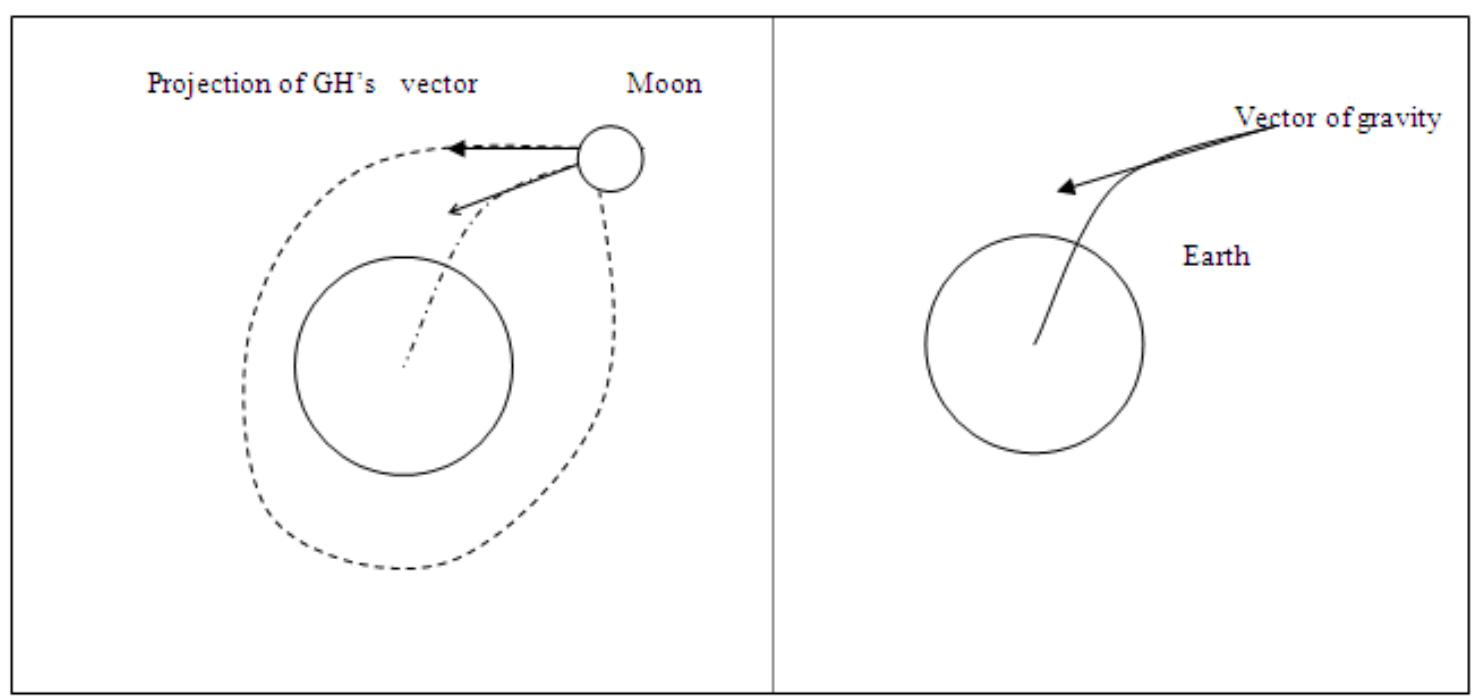

Figure 13. The projection of the vect or GH during the concentration of the earth's particles 


\section{The Formation of the Moon}

By studying the consequence of the convergence of particles that move in a helical form, we are able to explain the process in which the moon was formed.

The moon is considered as a satellite that turns around the earth, on the other hand it has similarities with objects that are fixed on the earth under the effect of gravity, because, like the moon, those objects turn around the earth and the sun, which means that, before the creation of the moon, its particles were part of the earth, this concept can provide us the hypothesis that the moon's formation was the consequence of the projection of a certa in amount of earth's particles in space, and during their return to the earth at curved speed of gravity they had converged into one single point which led to several consequences :

$\rightarrow$ The decrease of the speed of gravity GH

$\rightarrow$ The concentration of earth's particles which formed a spherical material because of the gravity's curvature that created a swirl of earth's particles before their collision into the earth.

$\rightarrow$ The enhancement of the rotation's diameter of the gravity's curvature caused by the projection of GH's vector which is the origin of the moon's rotation around the earth.

The diameter of the moon's rotation is proportional to the mass of the moon, and inversely proportional to its rotational speed.

\section{The Application of the Function 3 in Mechanical Quantum ( Infinitely Small)}

If we apply the concept stated in the paragraph 9 in quantum mechanics, we would deduce that electrons were formed by the same mechanism, which means the they are the consequence of the projection and the convergence of atomic partic les during the conversion of the light's energy into the mass ( Big Bang):

but when atoms were submitted to another swirl to forma bigger spherical mass that participated in the formation of the earth, It induced the immobilization of atoms because they are submitted to the effect of gravity which is not the case when it comes to electrons because if we study the figure above we would realize that when the atom is slowed down, the electron leaves the orbit in a spiral motion in the direction of the sphere's rotation (from west to east) and if we project this spiral movement on a plan we would get a wave motion that had been demonstrated by Louis de Brogolie.

On the other hand, atoms and electrons are endowed with the swirl of gravity, which means they are able to maintain small particles fixed on their surfaces if the diameter of the helical motions of those particles is identical to the diameter of the gravity's curvature (GH) of the electrons.

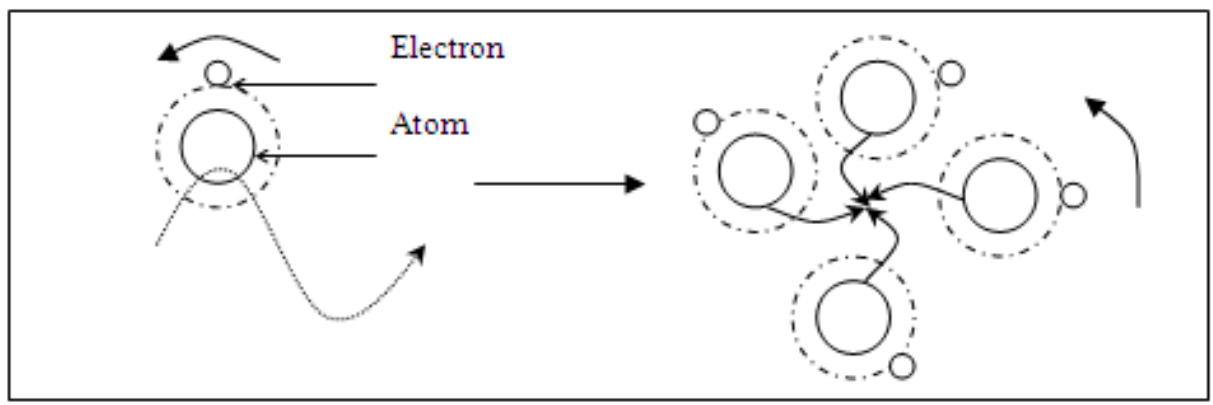

Figure 14. The concentration of at oms leads to the liberat ion of electrons

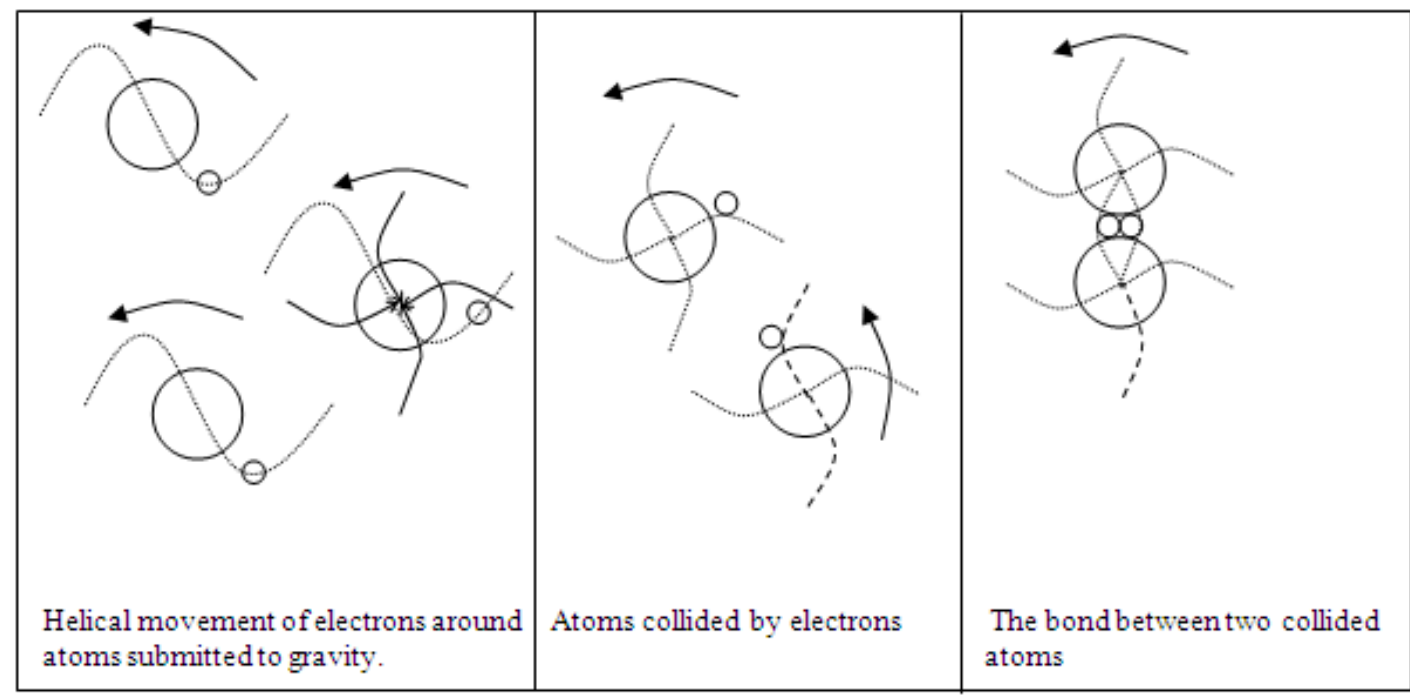

Figure 15. The formation of molecules after the fix ation of electrons by atoms 
To this end, electrons are able to absorb beams of light only if their helix's diameter matches with the electrons' gravity, and the main consequence of the light's absorption by electrons is the slowdown of their speed and the enhancement of their helical diameter, which means that when this diameter is higher than the curvature's diameter of the atom's gravity then electrons can rotate around the atom with a helical movement, but when the diameter of the electron's helix matches with the curvature of the atom's gravity then the electron collides into the atom which makes the atom likely to form bonds with other collided atoms in order to form molecules rotating around the center of the bond :

\section{Conclusions}

This article clarifies the mechanis $m$ of the conversion of the light's energy into the mass during the Big Bang where materials existed as photons that move at a high speed in space as a helical motion, and the synthes is of the matter started with a series of collisions between those photons into one single point, leading to the appearance of a spherical matter that rotates around its axis as well as the helix's axis, the resulting spheres are likely to collide with each other until the annulment of their linear speed which corresponds to an infinite mass of photons, in other words, the process of concentration turn the photons from small spheres (atoms) to gigantic spheres (planets)

From a mathematical point of view, the transition from one photon (infinitely small) to a big globe (infinitely great) is expressed by the function $M=1 / 1-\alpha$ which indicates that the linear speed is inversely proportional to the mass, on the other hand the photon's energy $\mathbf{E}=\mathbf{C} \cdot \mathbf{C o s} \sigma / \lambda$ ( related to Planck's formula $: E=h . C / \lambda)$ is converted to globe's energy $\mathbf{E}$ $=\mathbf{M} \cdot(\mathbf{C} \cdot \mathbf{C o s} \sigma)^{2} /$ GL. $\lambda$ ( related to Einstein's formula $\mathrm{E}$ $\left.=\mathrm{m} \cdot \mathrm{c}^{2}\right)$.

Also, gravity is the consequence of a swirl of particles (noticed by an immobile observer in space) that converge to one single point which reflects the center of gravity, and the swirl's characteristic of those spherical materials explain how electrons, atoms and planets are able to maintain particles fixed on their surfaces, we can also state that gravity is the driving force of spheres' rotation.

During the transition from infinitely s mall to infinitely great, the spiral beam of light is converted to a big circle in which planets rotate, this type of geometrical transformation is explained by the elevation of the tangent $\sigma$ and the annulment of $\lambda$ which closes the ellipsoidal helix and turns it into a ellipsoidal circle.

In the mechanical quantum, we deduce that electrons are not subject to the earth's gravity because they move in a helical tract in the direction of the earth's rotation, however when electrons absorb a certain amount of the light's energy, the diameter of their helical movement varies and can meet the diameter of the gravity's curvature of an atom, which means that electrons can be submitted to the atom's gravity which leads to the appearance of collided atoms that are able to form molecules.

The main conclusion we reach after studying this article, that is based on the concept of the concentration of photons into mass, is that Gravity is the consequence of the insolubility of Energy in space which led to the appearance of spherical materials endowed with periodic movements fro $m$ which had been established the notion of Time

\section{REFERENCES}

[1] The special relativity by Albert Einstein

[2] The General Relativity by Albert Einstein

[3] The formula of Max Planck: $\mathrm{E}=\mathrm{h} . \mathrm{C} / \lambda$

[4] The wave theory of light By Thomas Young

[5] The theory of Louis de Broglie concerning the movements of electrons as waves 